\title{
Magnetic configuration of a sunspot region using the $\mathbf{H} \beta$ line
}

\author{
Hongqi Zhang \\ Beijing Astronomical Observatory, Chinese Academy of Sciences Beijing 100080, China \\ Received June 6, 1995; accepted February 12, 1996
}

\begin{abstract}
The configuration of the magnetic field in an active region is analysed using a set of $\mathrm{H} \beta$ chromospheric magnetograms and narrow pass-band filtergrams taken at various positions on the line. We found that the chromospheric magnetic field is parallel to the chromospheric features and inhomogeneous. The photospheric magnetic field disappears abruptly in the edge of the penumbra; the chromospheric field extends beyond the penumbral boundary with an inclination of about $10^{\circ}-20^{\circ}$ to the solar surface. Chromospheric superpenumbral filaments show a good correlation with the chromospheric magnetic field and their configurations are related to the photospheric magnetic structures nearby the sunspot. Part of the magnetic flux of the sunspot returns to the neighbouring enhanced networks, which is almost vertical to the solar surface.
\end{abstract}

Key words: Sun: magnetic fields; chromosphere; sunspots

\section{Introduction}

Most models of the configuration of the magnetic field in solar active regions were established using observations of the photospheric magnetic field and their extrapolation. The configuration of the magnetic field also can be deduced from magnetograms obtained in spectral lines formed in different solar atmospheric layers. Measurements of the chromospheric magnetic field were made by Severny \& Bumba (1958), Giovanelli (1980), Giovanelli \& Jones (1982), Zhang et al. (1991 and 1993), Dara et al. (1993). The influence of the photospheric blended lines in the wing of the $\mathrm{H} \beta$ line for measurements of the chromospheric magnetic field has been analyzed by Zhang (1993). Analyses of the chromospheric magnetic field are few relative to photospheric ones, since most chromospheric lines are not highly sensitive to the magnetic field and chromosphere departures from local thermodynamic equilibrium.

The preliminary analyses based on the measurement of the magnetic field at various wavelengths in the wing of a single chromospheric line, such as the $\mathrm{H} \beta$ line, were made by Zhang $(1993,1994)$. The band pass of the filter in the Huairou magnetograph is $0.12 \AA$. It is much narrow than the width of the $\mathrm{H} \beta$ line. As we analyse a series of chromospheric magnetograms and corresponding filtergrams obtained at the various wavelengths from the wing to the center of the chromospheric $\mathrm{H} \beta$ line, we can estimate the possible formation layers of the magnetic field and the relationship with the monochromatic images from the photosphere to chromosphere (Zhang 1996).
In this paper, we present the configuration of the magnetic field in the active region based on photospheric vector magnetograms and chromospheric magnetograms with different wavelengths in the $\mathrm{H} \beta$ line obtained at the Huairou Solar Observing Station of the Beijing Astronomical Observatory. In Sect. 2, we show the observational data. In Sect. 3, we analyse the formation of the $\mathrm{H} \beta$ line and the relationship with chromospheric magnetograms. In Sect. 4, we discuss the possible spatial configuration of the magnetic field in sunspots and enhanced networks.

\section{Observational data}

A series of chromospheric and photospheric filtergrams and corresponding magnetograms in an $\alpha \mathrm{p}$ active region were observed in the end of August 1993, at the Huairou Solar Observing Station of the Beijing Astronomical Observatory. The preliminary results of this active region were presented by Zhang (1994). Figure 1 shows a photospheric image and a photospheric magnetogram in this region (S3.2, W57) on August 27, 1993. We can find that the sunspot of this region is positive and a part of the magnetic field shows negative polarity on the west side of the sunspot, due to the projection effect of the magnetic field. Enhanced networks of negative polarity are located on the east side of the sunspot.

Figure 2 shows a set of $\mathrm{H} \beta$ filtergrams at different wavelengths with an interval of $0.04 \AA$, between $-0.40 \AA$ and $-0.12 \AA$ from the line center. In the filtergram at $\mathrm{H} \beta-0.40 \AA$, the chromospheric features are not distinct. It is similar to the photospheric image. We 

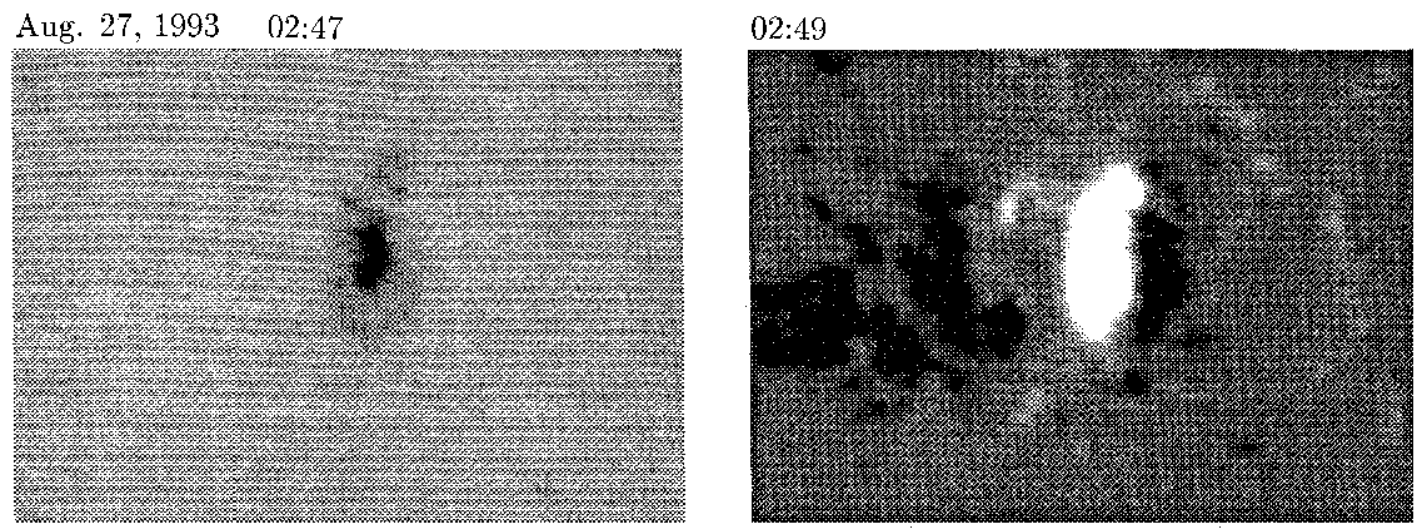

Fig. 1. A unipolar sunspot region on Aug. 27, 1993. A photospheric image (left) and a longitudinal magnetogram (right), white (black) is positive (negative) polarity. North is at the top, west is at the right

notice that as the observed wavelength in the set of filtergrams approaches the $\mathrm{H} \beta$ line center, chromospheric features become significant. The dark filaments extend and their lengths increase from outside the sunspot penumbra in the west of the sunspot. These filaments constitute the superpenumbral features. On the east side of the sunspot, there is no evidence of filament-like features. It is possible that the spatial resolution of these images is lower, so chromospheric fine features cannot be found.

Figure 3 shows a set of chromospheric longitudinal magnetograms. Filament-like magnetic features in the magnetograms show good correlation with features in the chromospheric filtergrams. The lengths of chromospheric magnetic filament-like features $f_{1}$ and $f_{2}$ increase with dark filaments as observed wavelengths shift to the $\mathrm{H} \beta$ line center. These chromospheric features thus reflect information on the magnetic field formed in the corresponding atmospheric layers.

\section{Data analysis}

The formation of the $\mathrm{H} \beta$ line in the chromospheric magnetic field was analysed by Zhang (1996). The emergent light of $\mathrm{H} \beta$ Stokes parameters mainly comes from both layers, chromosphere and photosphere, but there is little contribution from the temperature minimum zone. The emergent Stokes parameters near the $\mathrm{H} \beta$ line center form mainly in the chromospheric layer and those in the wing form in the photospheric layer. As the observed wavelength of the Huairou Magnetograph shifts from the wing of the $\mathrm{H} \beta$ line to the line center, the emergent light formed in the photosphere decreases gradually and that formed in the chromosphere increases. From magnetograms observed at various wavelengths from the wing of the line to the center, we can estimate the possible configuration of the magnetic field.

The real formation layers of Stokes parameters of the $\mathrm{H} \beta$ line are more complex. The formation heights of dif- ferent kinds of solar structures observed in the $\mathrm{H} \beta$ images may be different, such as sunspot umbrae, penumbrae and dark filaments, even though these features are observed at the same wavelength in the wing of the chromospheric line.

The disturbance of photospheric blended lines in the wing of the $\mathrm{H} \beta$ line is significant for the measurement of the magnetic field in sunspot umbrae. The evident influence of the blended line FeI $\lambda 4860.98 \AA$ in the $V$ profile of the umbra was discussed by Zhang (1996). The reversal structures relative to the photospheric field can be found in the $\mathrm{H} \beta$ chromospheric magnetograms near such wavelengths. The influence of the chromospheric mass motion is a notable problem for the measurement of the chromospheric magnetic field. The Doppler motion of chromospheric mass shifts the line. If the reversal structures in chromospheric magnetograms are only caused by the mass motion, we should obtain these reversal structures at a series of wavelengths in the wing of the shifted $\mathrm{H} \beta$ line. This is not consistent with our results presented above. However, due to the violent relative motion between the photospheric and chromospheric masses in the sunspot umbrae, the disturbance of the photospheric blended lines probably becomes more significant.

From a series of magnetograms obtained at various wavelengths in the wing of the wide chromospheric lines, such as $\mathrm{H} \alpha$ and $\mathrm{H} \beta$ lines, we can understand the gradual change of the magnetic field from the photosphere to the chromosphere, except in sunspot umbrae, where the disturbance of photospheric blended lines is significant. We notice that the magnetic sensitivity of the line changes with wavelength. For example, near the $\mathrm{H} \beta$ line center the sensitivity is weaker than that in the wing of the line. 
$-0.40 \AA$

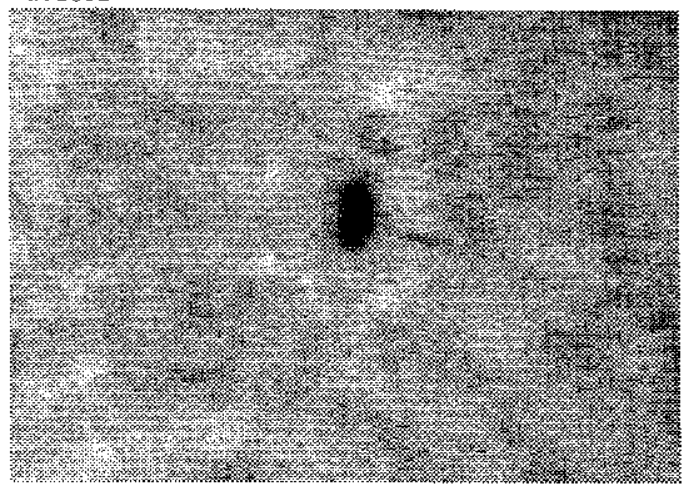

$-0.32 \AA$

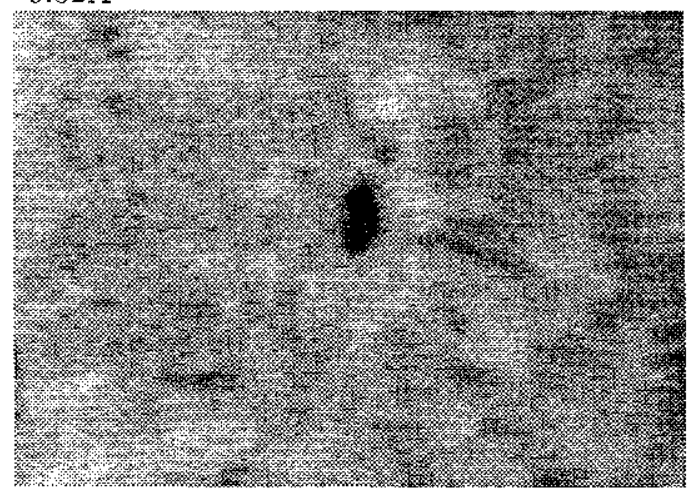

$-0.24 \AA$

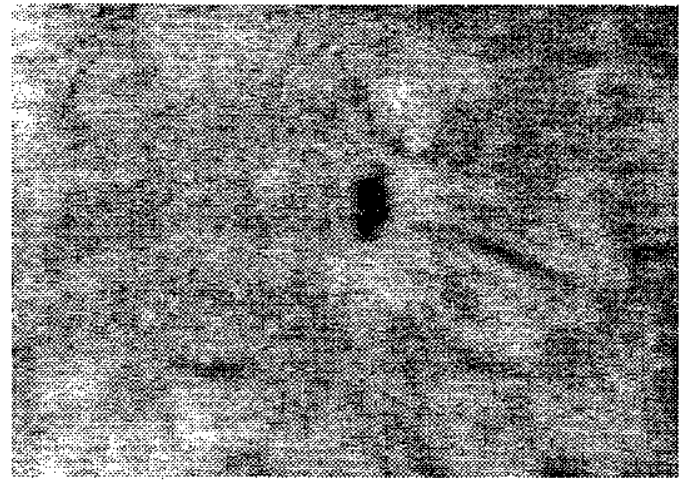

$-0.16 \AA$

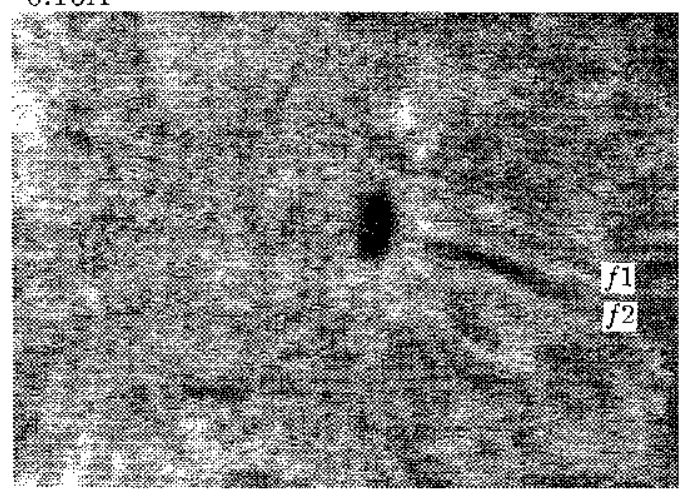

$-0.36 \AA$

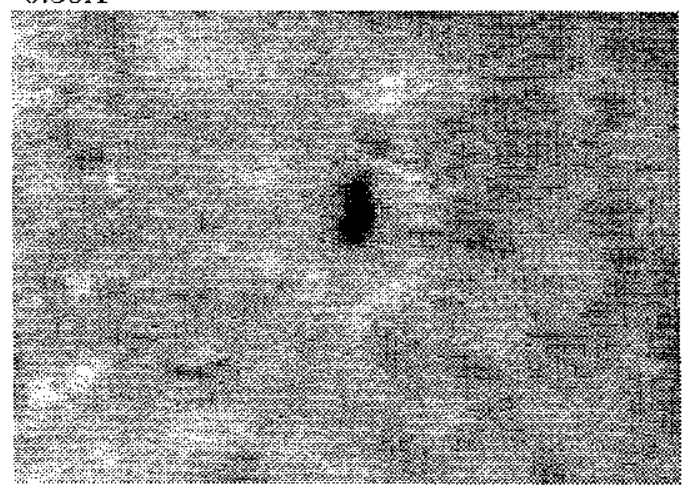

$-0.28 \AA$
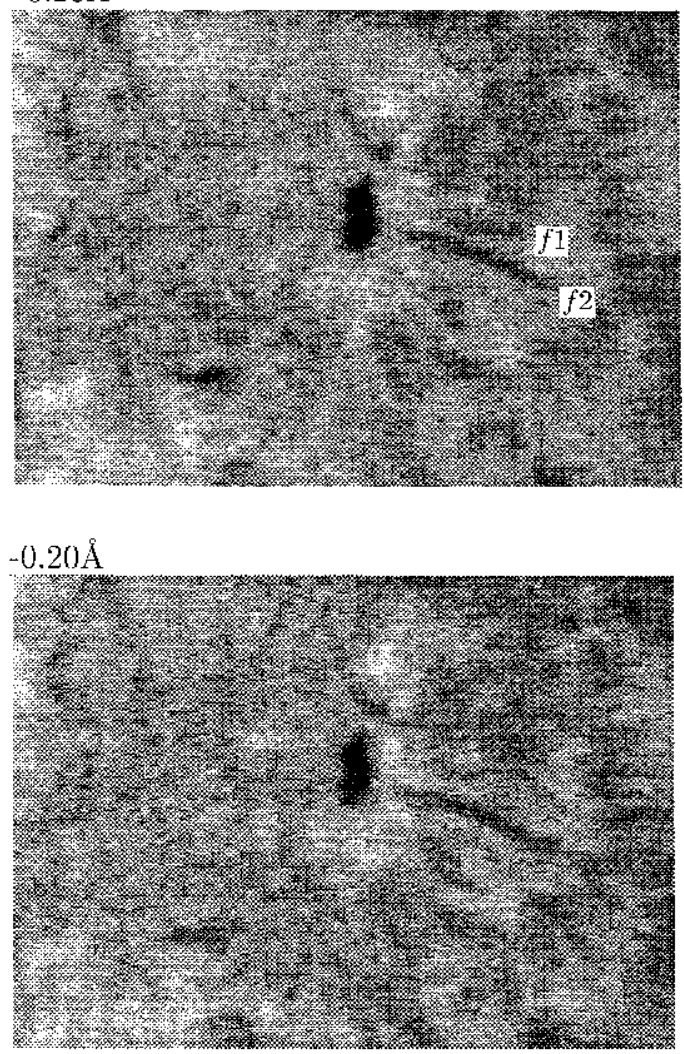

$-0.12 \AA$

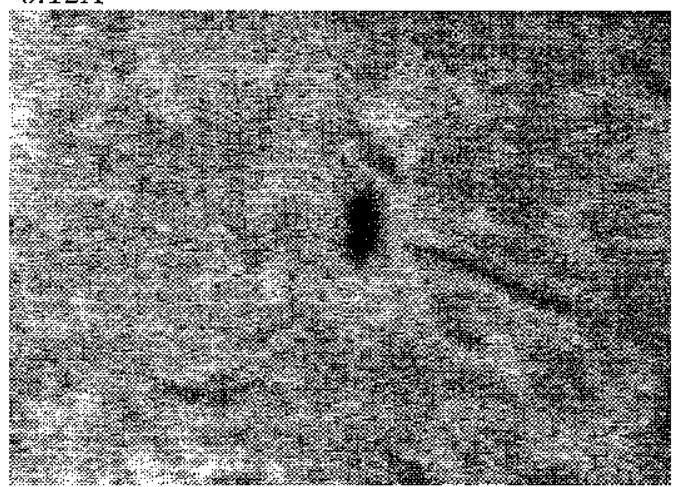

Fig. 2. A set of chromospheric filtergrams of the unipolar sunspot region at wavelengths $-0.40,-0.36,-0.32,-0.28,-0.24$, $-0.20,-0.16$ and $-0.12 \AA$ from the $\mathrm{H} \beta$ line center at $0258-0313 \mathrm{UT}$ on Aug. 27, 1993 

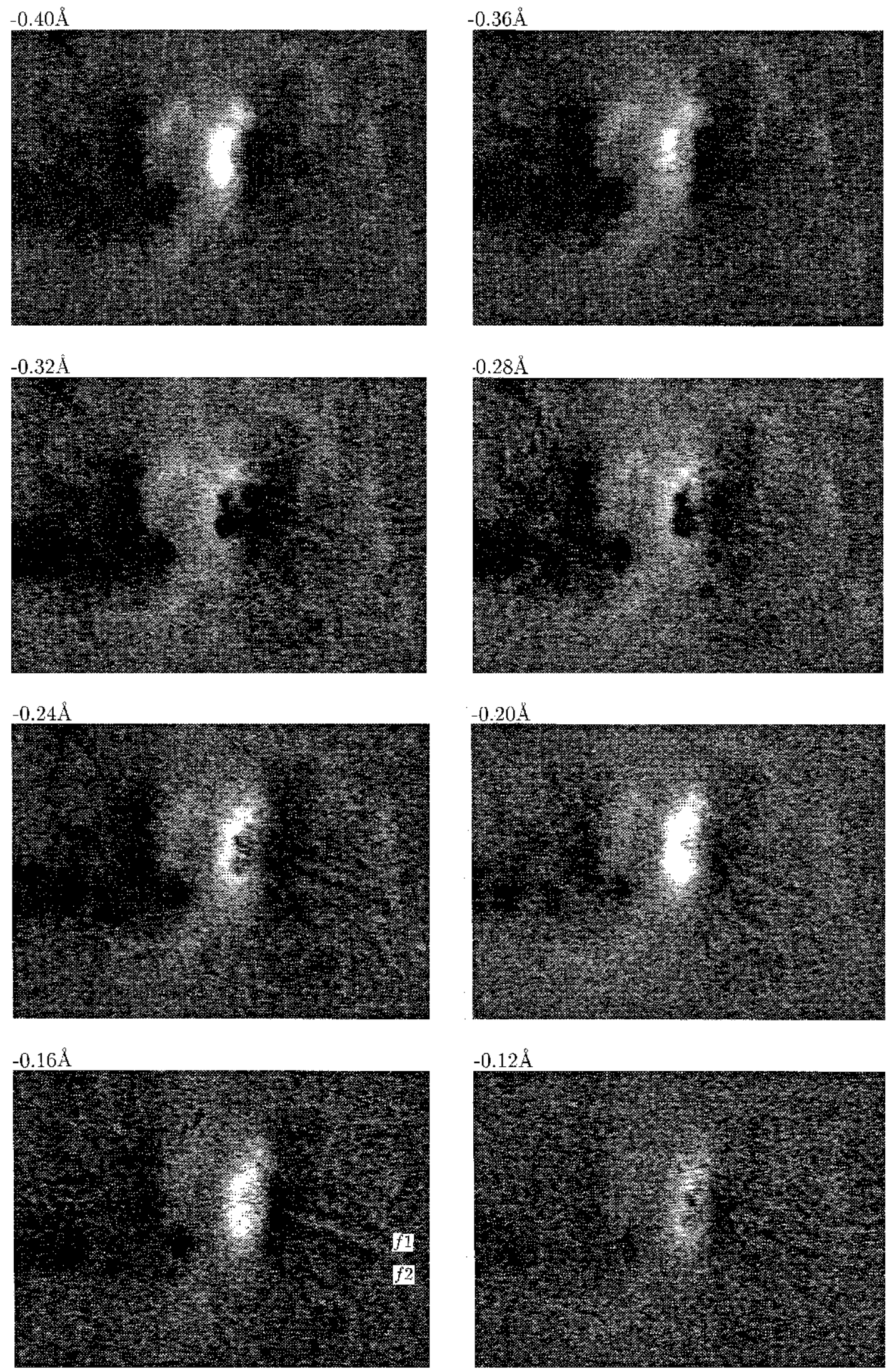

Fig. 3. A set of chromospheric longitudinal magnetograms of the unipolar sunspot region at wavelengths $-0.40,-0.36,-0.32$, $-0.28,-0.24,-0.20,-0.16$ and $-0.12 \AA$ from the $\mathrm{H} \beta$ line center at $0258-0313 \mathrm{UT}$ on Aug. 27, 1993. White (black) is positive (negative) polarity 


\section{Configuration of the chromospheric magnetic field}

\subsection{Perspective effect of the magnetic field}

Since the active region on August 27, 1993 was located near the west limb of the solar disk (S3.2, W57), the angle between the longitudinal and the horizontal magnetic fields (on the solar surface) is about $30^{\circ}$ in the plane which contains the line-of-sight and the normal to the solar surface. This means that some features in the longitudinal magnetograms reflect mainly information on the horizontal components of the magnetic field, while the transversal components of the magnetic field reflect mainly the vertical ones relative to the solar surface. Figure 4 shows a photospheric vector magnetogram in the active region. The pattern in the chromospheric magnetograms obtained at $\mathrm{H} \beta-0.40 \AA$ is similar to the photospheric one. A set of chromospheric magnetograms obtained at various wavelengths from the wing of the $\mathrm{H} \beta$ line to the center can be used to infer the variation of the line-of-sight components of the magnetic field with height in the chromosphere.

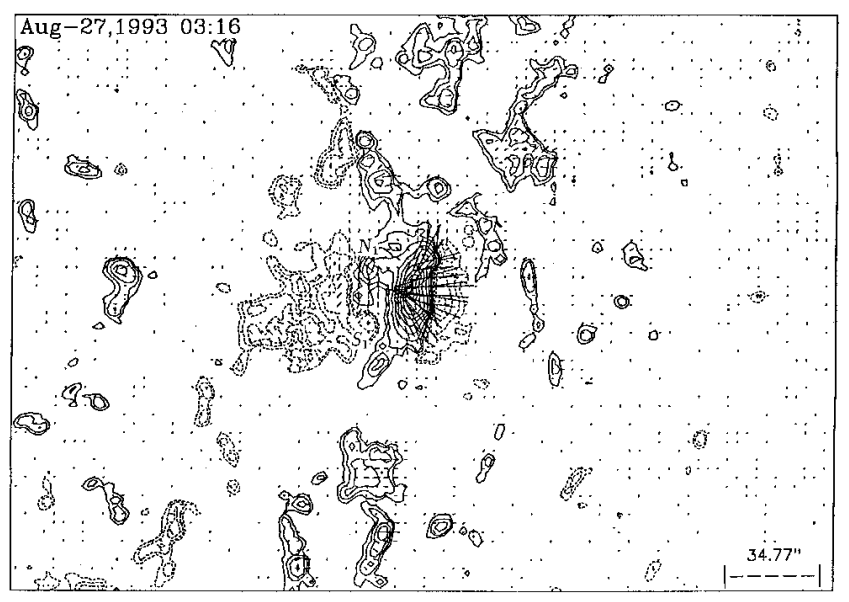

Fig. 4. A vector photospheric magnetogram of the unipolar sunspot region on Aug. 27, 1993. The solid (dashed) contours correspond to positive (negative) fields of 20,40, 80, 160, 320, 640, 960, 1280 Gauss

\subsection{Inclination angles of the superpenumbral magnetic field}

The observational results show that the magnetic field diffuses from the sunspot. The question is how do the magnetic lines of force incline to the solar surface in or beyond the edge of the sunspot penumbrae. Different results were obtained by various authors (e.g. Giovanelli 1982; Schmidt et al. 1986; Jahn 1989; Solanki \& Schmidt 1993). Figure 4 shows the photospheric vector magnetogram in the active region. The transversal magnetic field extends from the sunspot center and connects with a magnetic structure of opposite polarity on the west side of the sunspot, due to the projection effect of the magnetic field. In the west side of the sunspot, the photospheric magnetic field disappears abruptly at the edge of the penumbra, while chromospheric magnetic features exhibit rapid spreading from the spot with no evidence that the magnetic flux returns to the neighbouring region as the observed wavelength goes to the $\mathrm{H} \beta$ line center. If the $\mathrm{H} \beta$ line center forms at $1600 \mathrm{~km}$ (Zhang 1996) and comparing with the photospheric vector magnetogram, the inclination angle of the magnetic field at the boundary of the sunspot is about $10^{\circ}-20^{\circ}$ relative to the solar surface, from the superpenumbral interior boundary to the outer one.

On the east side of the sunspot, the chromospheric magnetic neutral line is close to the sunspot, and it does not show any significant shift with height. The photospheric transversal magnetic field connects the magnetic features $N_{1}$ and $S_{1}$ of opposite polarity. There are no evident superpenumbral features. We can infer that the magnetic lines of force bridge over the magnetic neutral line. The magnetic lines of force come from the sunspot and return to the solar surface near the outer edge of the sunspot. The magnetic field takes the return-flux model (Osherovich 1982).

\subsection{Magnetic field in enhanced networks}

Comparing with the photospheric vector magnetogram (Fig. 4) we find that the noise of the chromospheric magnetograms is higher than the photospheric ones. The fine features of the magnetic networks are not distinct. Since this active region was located near the solar limb, the network magnetic field shows evident transversal components in the photospheric vector magnetogram. The chromospheric magnetic features of enhanced networks on the east side of the sunspot exhibit almost the same pattern at different wavelengths from the wing to the center of the $\mathrm{H} \beta$ line. The reason is that, in the chromosphere, the large-scale network magnetic field near this active region does not contain an obvious horizontal component. Therefore, the large-scale enhanced network magnetic field near this active region is almost vertical to the surface. Even the small-scale magnetic features in networks consist of fibril-like magnetic features as demonstrated by Zhang et al. (1991). Zhang et al. indicated that some magnetic lines of force extend from the network magnetic elements to the upper solar atmosphere and some lines of force return to the neighbouring regions in the lower atmosphere.

\subsection{Correlation between chromospheric features and magnetic field}

In comparison of the chromospheric longitudinal magnetograms with corresponding filtergrams, we find that, as the observational wavelengths change from the wing of the $\mathrm{H} \beta$ line to the center, the pattern in chromospheric magnetograms gradually departs from the photospheric one, 
and chromospheric filament-like magnetic features appear. The superpenumbral dark filaments show a good relationship with the chromospheric magnetic features, e.g. in the chromosphere, dark superpenumbral features show a stronger magnetic horizontal field. The correlation between the magnetic field and the monochromatic images in the chromosphere is better than the photospheric ones.

\section{Discussion}

It is evident that the spatial configuration of the chromospheric magnetic field in the sunspot region is connected with the surrounding magnetic structures observed in the chromospheric magnetograms. The chromospheric superpenumbral magnetic field on the west side of the sunspot is shallow and changes on both sides of the boundary surface of the superpenumbra relative to the surrounding atmosphere. The opposite case can also be found. A part of penumbral magnetic flux on the east side of the sunspot returns to the enhanced network near the boundary of the sunspot and forms a return-flux model. This means that the extension of the magnetic flux from the sunspot is complex. A similar situation was also discussed by Zhang et al. (1991) from chromospheric $\mathrm{H} \beta$ magnetograms and $\mathrm{H} \alpha$ filtergrams. The magnetic canopy model (Giovanelli 1982) and the return-flux model (Osherovich 1982; Flå et al. 1982) also reflect the distribution of the sunspot magnetic field.

Moreover, since the magnetic field chenges obviously on both sides of the superpenumbral boundary surface the annular electric current probably forms in the sunspot penumbral interface to the non-magnetic atmosphere.

\section{Summary}

Following analyses of the configuration of the field in an $\alpha p$ active region with a series of chromospheric filtergrams and corresponding chromospheric magnetograms obtained at different wavelengths in the $\mathrm{H} \beta$ line, the main results are:

(1) The distribution of magnetic fields in different solar atmospheric layers can be obtained with a single chromospheric line, such as the $\mathrm{H} \beta$ line, because emergent Stokes parameters in the $\mathrm{H} \beta$ line wing form mainly in the lower solar atmosphere while the emergent Stokes parameters near the $\mathrm{H} \beta$ line center form mainly in the higher atmosphere.
(2) A good correlation between sunspot superpenumbral dark filaments and chromospheric magnetic features is found. As the observational wavelength changes from the wing of the $\mathrm{H} \beta$ line to the center, changes of superpenumbral dark filaments accompany the increase of the chromospheric filament-like magnetic features, e.g. chromospheric features reflect the distribution of the magnetic field. The chromospheric filament-like magnetic field extends at small angles to the solar surface.

(3) The extension of the sunspot chromospheric magnetic field depends on the distribution of the surrounding photospheric magnetic field. In our presented active region, a part of the sunspot magnetic flux returns to the neighbouring enhanced networks consistent with the return-flux model.

(4) In enhanced networks of this active region, the large-scale magnetic field is almost vertical to the solar surface and connects with the neighbouring sunspot field.

Acknowledgements. The author is grateful to staff at Huairou Solar Observing Station for their support in these observations. He also thanks Dr. S. Koutchmy for helpful comments and suggestions. This research was supported by the Chinese Academy of Sciences and National Science Foundation of China.

\section{References}

Dara H.C., Koutchmy S., Alissandrakis C.E., 1993, A\&A 277, 648

Flå T., Osherovich V.A., Skumanich A., 1982, ApJ 261, 700

Giovanelli R.G., 1980, Solar Phys. 68, 49

Giovanelli R.G., Jones H.P., 1982, Solar Phys. 79, 267

Jahn K., 1989, A\&A 222, 264

Osherovich V.A., 1982, Solar Phys. 77, 63

Schmidt H.U., Spruit H.C., Weiss N.O., 1986, A\&A 158, 351

Severny A.B., Bumba V., 1958, Observatory 78, 33

Solanki S.K., Schmidt H.U., 1993, A\&A 158, 351

Zhang H., 1993, Solar Phys. 146, 75

Zhang H., 1994, Solar Phys. 154, 207

Zhang H., 1996, (Submitted to A\&A)

Zhang H., Ai G., Sakurai T., Kurokawa H., 1991, Solar Phys. 136, 269

Zhang H., Ai G., Li W., Chen J., 1993, Solar Phys. 146, 61 